\title{
LA VERDADERA HISTORIA DE DON PEDRO GONZALES, EL «HOMBRE SALVAJE» DE TENERIFE QUE LLEGÓ A SER PROFESOR DE LA SORBONA DE PARÍS
}

\author{
Alberto Quartapelle* \\ cronicascanarias@gmail.com
}

\section{RESUMEN}

Don Pedro Gonzales, el hombre salvaje, como fue apodado por sus contemporáneos, padecía hipertricosis, una rara enfermedad que le causaba un crecimiento incontrolable del pelo de la cara y de todo el cuerpo. Hasta ahora, se conocía muy poco de su vida, sobre todo de su permanencia en la corte de los reyes de Francia. Nuevos documentos encontrados en los archivos franceses demuestran que, a pesar de su enfermedad, no solamente logró vivir una vida normal con su esposa y sus hijos, sino que llegó a ser un influyente miembro de su comunidad y de la corte.

Palabras clave: don Pedro Gonzales, hombre-lobo, hombre salvaje, hipertricosis.

\section{THE TRUE STORY OF DON PEDRO GONZALES, THE “WILD MAN” FROM TENERIFE WHO BECAME A PROFESSOR AT THE SORBONNE IN PARIS}

\section{Abstract}

Don Pedro Gonzales, the "wild man," as he was nicknamed by his contemporaries, suffered from hypertrichosis, a rare disease that caused uncontrollable growth of hair on his face and all over his body. Until now, truly little was known about his life, especially about his stay at the court of the kings of France. New documents found in French archives show that despite his illness, he not only managed to lead a normal life with his wife and children, but also became an influential member of his community and of the court.

Keywords: Don Pedro Gonzales, Wild Man, Wolfman, Hypertrichosis. 


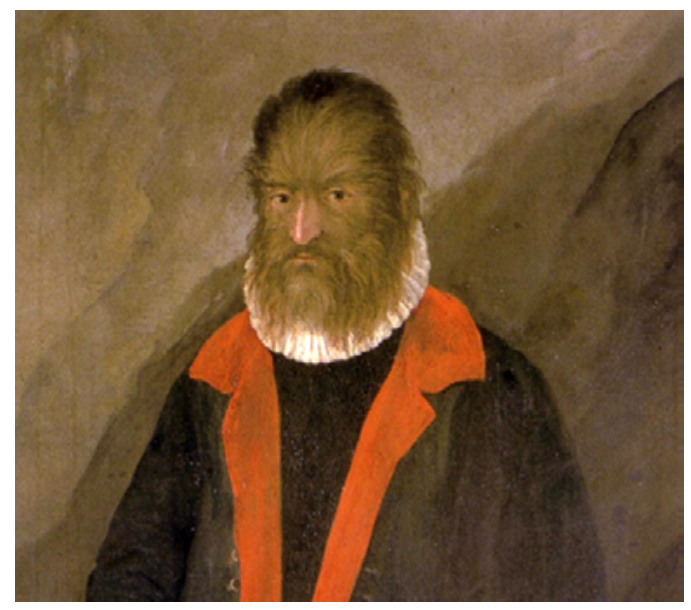

Fig. 1: Petrus Gonsalvus: Cámara de Arte y Curiosidades Castillo Ambras (Innsbruck-11 Austria).

\section{INTRODUCCIÓN}

El 18 de abril de 1547 llegaba a la corte del rey de Francia Enrique II, un niño cuya cara y cuerpo estaban cubiertos de pelos. Su singular aspecto se debía a una rara enfermedad congénita que todavía era desconocida en esa época, la hipertricosis, la cual causa un crecimiento incontrolable del pelo.

Objeto a veces de estupor y otras de morbosa curiosidad, este hombre nativo de la isla de Tenerife, cuyo nombre era Pedro Gonzales, fue apodado como «salvaje» o como «hombre-lobo». Este estigma lo acompañó toda su vida y, por ello, tuvo que combatir contra los prejuicios de sus contemporáneos.

Mucho se ha escrito acerca de este personaje, sin embargo, cuando se leen las biografías de don Pedro ${ }^{1}$, como siempre quiso que lo llamaran, lo primero que se destaca es que los autores dedican muchas páginas a describir el mundo en el que vivió y pocas a su historia personal. Los verdaderos protagonistas de esos libros son reyes y reinas, enanos, locos y bufones de la corte, duques y cardenales, pintores y médicos, mientras que don Pedro es solo una sombra que se mueve en segundo plano.

El motivo de esta extraña situación se debe a que, hasta ahora, para reconstruir su vida los biógrafos disponían solo de unos pocos documentos y de algunos

* https://independent.academia.edu/AQuartapelle.

1 Zapperi, R.: Il selvaggio gentiluomo, Donzelli, Ed., Roma (2005); Carrasco, E.: Gonsalvus. Mi vida entre lobos, Ediciones IDEA, Santa Cruz de Tenerife, (2006); Bouillot, I.: Le sauvage du roi, (2015); Ghadessi, T.: Portrait of human monsters in the Ranaissance, Western Michigan Univ. Medieval, (2018); Wiesner-Hanks Merry, E.: The Marvelous Hairy Girls; The Gonzales Sisters and Their Worlds, Yale University Press, (2009). 
retratos de sus hijos, "peludos» como él, que solo permitían hacer suposiciones acerca de su vida, algunas veces acertadas y otras sin fundamento.

Treinta nuevos documentos (anexo 1) procedentes de los archivos franceses, memorias, actas notariales, certificados de bautismo, nos ofrecen una nueva perspectiva de la vida de este extraño personaje que, por sus cualidades, no fue considerado por sus contemporáneos ni un «monstruo» ni una broma de la naturaleza, sino un hombre capaz de integrarse perfectamente en la sociedad en la que vivió. El nuevo retrato que aquí se propone es el de un hombre que no solamente logró superar su enfermedad, sino que ayudó a modificar la concepción de "diversidad» que se tenía en su tiempo.

En el artículo no se habla de sus hijos, algunos de los cuales no tuvieron la suerte del padre y, para poder vivir, fueron obligados a aceptar ser exhibidos frente a la curiosidad general. La única excepción será la publicación de un retrato de una de las hijas de Pedro (anexo 2), hasta ahora no publicado, que pertenece a una colección privada.

\section{EL JOVEN PEDRO (1547-1560)}

Lo poco que conocemos de la llegada de Pedro Gonzales a la corte del rey de Francia, Enrique II, se lo debemos a una carta enviada por el embajador Giulio Alvarotto el 18 de abril de 1547 al Duque de Ferrara Ercole d'Este':

... han donado al rey (de Francia Enrique II) un niño de alrededor de x anos traído desde las Indias, muy lindo, pero tiene la cara y el cuerpo todo peludo, como por cierto se dice sean los hombres salvajes. Los pelos son largos cinco dedos. Son muy ralos, tanto que se pueden ver todos los rasgos de su cara. Son color castaño claro y más finos que los de la marta y tienen un buen olor. Habla espańol y viste como todos los demás. Pero en el cuerpo y las espaldas tiene el pelo maltrecho. No sé quién lo donó a Su Majestad.

2 «... È stato donato al re un putto de circa X anni portato dalle Indie, molto bello, ma tutto piloso il volto et tutta la vita, come appunto si dipingono gl'humani silvatici. I pelli sonno longhi circa cinque dita. Sonno rari molto, tanto che si vedeno tutti i lineamenti della fazza. Sonno di colore tané chiaro et molto sottili et fini più che'l pello di zebelino, et sanno de buon. Lui parla spagnuolo et va vestito come è l'ordinario d'ognuno. Però su per la vita ha il pello frusto. Non si chi l'habbi donato a Sua Maestà», 'Archivio di Stato di Modena, Archivio segreto estense, Cancelleria ducale, Sezione estero, Carteggio ambasciatori, Francia, b. 24. En ZapperI, R. op. cit. p. 158. Otros médicos de la época dedican unas líneas a Pedro: el italiano Realdo Colombo, que en realidad nunca pudo verlo, escribe: "He visto también un español cubierto de pelos en todo el cuerpo y también en la cara y en las manos" - "Ego tamen hispanum quendam vidi pilis refertissimum in omni corporis parte praeterquam in facie \& mannum parte» en "De re anatomica libri XV» 1559-Liber XIII, cap. II p. 255. Y Johannes-Lonaeus Bosch en 1582: "El rey de Francia Enrique II se preocupó de instruir en los conocimientos humanos un hombre no menos piloso de un perro». "Rex Galliarum Henricus Lutetiae Parisiorum literis humanioribus instituendum curauit hominem cane non minus villosum» en "Concordia medicorum et physicorum de humano conceptu, atque foetus...» cap. XLI. 
Pocos años después el médico italiano Cesare Scaligero, en su «Exoticarum exercitationum liber XV», añade nuevas informaciones acerca del joven: «... El muchacho está totalmente cubierto de pelo blanco. Por este motivo, los franceses lo llamaron Barbet. Con este nombre se referían a un perro de pelo erizado que en Flandes llaman Watterhund $»^{3}$.

Hasta ahora se suponía que la llegada de Pedro a Francia se debía a alguna incursión de corsarios franceses en las costas de las Islas Canarias para apoderarse de esclavos.

Sin embargo, el embajador Alvarotto, quien como era frecuente en Italia en el siglo Xvi confundía las Islas Canarias con las Indias, ubicó a España como lugar de procedencia de Pedro. Esta circunstancia sugiere que el joven era el mismo niño velludo descrito por Antonio de Torquemada en el Jardin de flores curiosas:

Y en nuestros tiempos, porque puede haber veinte ańos (desde 1570), poco más o menos, andaba un hombre por toda España mostrando un hijo suyo y sacando dineros con él: el muchacho tenía diez u once años y era cosa, cierto, para ver, porque su vello era tanto y tan largo y espeso, que en la cara no se le parecía sino la boca y los ojos, y estaba este vello tan encrespado que hacía unas como sortijas; y verdaderamente los salvajes que pintan no están tan disformes ni cubiertos en todo el cuerpo, como este muchacho lo estaba ${ }^{4}$.

Si se considera que se registra menos de un caso de hipertricosis por siglo, esta coincidencia sugiere que después de haber sacado provecho de la exhibición del cuerpo peludo de su hijo en toda Espańa, el padre lo haya vendido al rey de Francia.

La venta del niño «salvaje» a Enrique II no debe sorprender. Enanos, gigantes, jorobados, locos y bufones eran figuras habituales en las cortes renacentistas. Los soberanos de la época amaban estar rodeados de todo tipo de seres «no normales» para su diversión. Estos sujetos ofrecían el contraste necesario frente a la prudencia, la majestad, la belleza y la compostura que representaban sus señores 5 .

Sin embargo, convertir al nińo peludo en un entretenimiento para los cortesanos no era el destino que le había reservado su nuevo amo. Así, poco después de su llegada a la corte, en 1548, el rey lo puso bajo la protección de un gouverneur, quien tenía que brindarle cuidados e instrucción. Por cierto, la designación de un gouverneur para atender al joven babet no era un hecho excepcional, ya que los había también para los leones que vivían en el parque de l'Hotel des Tournelle. Menos normal era que el rey escogiera para este encargo a Barthélemy Vallère, un

3 «De puero piloso. Exigit locus hic historiam de Hispano puero : quem ex India advectum, alij parentibus Indicis in Hispania natum putant. Is candidis pilis totus tectus fuit. Iccirco Barbet à Gallis dictus. Quo nomine villosum vocant canem, quem Flandri Watterhund». Iulii Caesaris Scaligeri: «Exoticarum exercitationum liber XV» (1557) cap. CxIv.2.

${ }^{4}$ Antonio de Torquemada: «Jardín de flores curiosas» (1570), ed. Enrique Suárez Figaredo-Lemir 16 (2012) p. 639. En Pentassuglia, P.: La reconnaissance de la diversité au XV siécle. A' propos de la difformité, ALTER, European Journal of Disability Research 5 (2012) 233-248.

5 Garcia Garcia, B.: Elocio en la España del Siglo de Oro, Ediciones AKAl (1999) pp. 33-34. 
importante cortesano, quien como "huisseur de la chambre du roi», era el primero que veía al soberano por la mańana y aquel que se encargaba del control de todas las persona que lo visitaban. Y tampoco era normal que Pedro fuese enviado a vivir con la familia de Pierre Hotman, rico y culto burgués, y uno de los más apreciados plateros de París ${ }^{6}$.

En 1551, el gouvernement du saulvaige du Roy, el «salvaje del rey», como desde este momento empezaría a llamarse Pedro, pasó a otro cortesano, Francois de la Vacherie, que lo tendría a cargo por lo menos hasta $1552^{7}$.

Pocos años después, la instrucción recibida y la protección de Enrique II, que quería demonstrar que se podía transformar un pequeño «salvaje» en un gentilhombre, le abrieron las puertas de la corte. En 1556, cuando tenía cerca de 20 años, y hasta 1560, encontramos a un Pierre Sauvaige entre los officiers domestiques $d u$ roy, con el cargo de sommelier de panneterie bouche, algo similar a un camarero que servía la mesa real ${ }^{8}$.

\section{EL MANTO DE SILENCIO (1560-1584)}

A partir de 1560, las biografías de Pedro Gonzales señalan un largo período oscuro de más de veinte años. Aparentemente sobre él y sus pelos cayó un silencio que permaneció por muchos años totalmente impenetrable. El motivo de lo que los biógrafos han llamado el «manto de silencio» se ha justificado aduciendo la preocupación de Pedro de hacerse notar lo menos posible, de pasar desapercibido, de no ser el centro de atención debido a sus pelos, sobre todo después de la muerte de su protector Enrique II, en $1559^{9}$.

En realidad, nuevos documentos aclaran aspectos desconocidos de su vida privada y pública, y demuestran que no solamente don Pedro no se escondió para

${ }^{6} 17$ de mayo de 1548: «Procuration de Barthélemy Vallère, huissier de salle du roi, et ayant la charge de la dépense et nourriture du' sauvage du roi', à Pierre Hotman l'aîné, marchand orfèvre, bourgeois de Paris, pour recevoir ce qui lui sera dû à cause de ladite dépense, à commencer le ler juin, en bailler quittance à André Blondet, trésorier de l'épargne». Notaire Jean TrouvéANFr MC/ET/XIX/175

7 «Procuration donnèe par Francois de la Vacherie ayant la charge et gouvernement du saulvaige du Roy, n[otre] s[ire] à Pierre le Moyen, pour recevoir de M. André Blondet, trésorier de l'Epargne du roi les deniers qui luy sont ordennez par led. Sgr pour les moys de juillet, aoust et septiembre prochains venans pour la nourriture et gouvernement et despence du saulvaige d'icellui Sgr, qui est à raison de 50 sols. Par chacun jour, et ce pour l'absence dud. Constiutuant, qui s'en va au roiaulme d'Angleterre par le commandement du Roy et pour ses affaires».

${ }^{8}$ ZAPPERI, R. op. cit., p. 39

9 Zapperi, R. op. cit., pp. 43-46. Como prueba de la existencia en la corte de París de una "conspiración de silencio» a protección de Pedro se ha recordado que Ambroise Paré, médico personal y frecuentador de la corte, en su libro De monstres et prodiges publicado en 1573 a pesar de describir de una mujer velluda no habla de él. Les oeuvres de M. Ambroise Paré conseiller, et premier chirurgien du Roy: libres des monstres et prodiges, Parigi (1595) chap. DV, p. 818. 


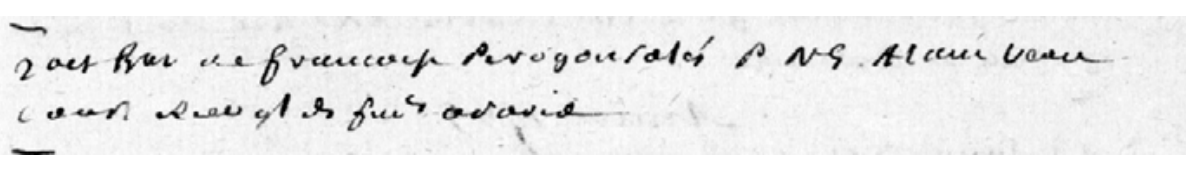

Fig. 2. «2 oct[obre] Bat (baptême) de francoise Perogonsalés P[arrain] N[oble] h[omme] Alain Veau cons[eille]r rece[veur] des fin[anc]es à Paris».

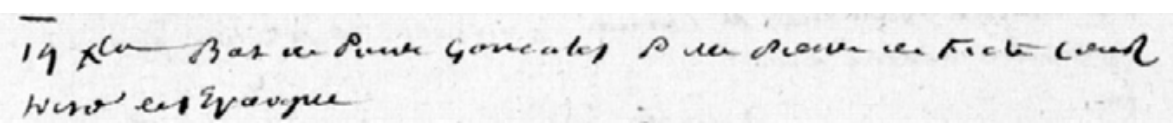

Fig. 3. «19 xbe (décembre) Bat (baptême) de Perre Goncales P[arrain] Me Pierre de Ficte Cons[eille]r treso[rier] de l'Epargne».

proteger su «diferencia», sino que ocupó posiciones relevantes en la corte y en la sociedad (anexo 1).

En lo que se refiere a su vida privada, se ha encontrado el contrato matrimonial estipulado en 1570 con su futura esposa Catherine y las actas de bautismo de cuatro de sus hijos, Francoise, Pierre, Henry y Charlotte, nacidos entre 1571 y 1578, datos que modifican notablemente lo que se había supuesto hasta el momento ${ }^{10}$ :

1570: se casa en París con una joven burguesa, Catherine Raffelin, hija de un mercante textil. De acuerdo con el contrato matrimonial y como era la costumbre, la esposa trae como dote la mitad de un apartamento en la rue Saint-Victoir, en París, donde el matrimonio se muda.

1571: el 2 de agosto, es bautizada Francoise, su primera hija, en la iglesia de SaintNicolas du Chardonnet. Como testimonio de la continuidad de la relación privilegiada entre Pedro y la corte incluso después de la muerte de su protector, el rey Enrique II, el padrino de la pequeña fue Alain Veau, responsable de las finanzas públicas del nuevo rey Carlos IX (fig. 2).

1572: el 2 de agosto es bautizado, siempre en la iglesia de Saint-Nicolas du Chardonnet, un hijo varón llamado Perre (¿Pierre?). El padrino en este caso es Pierre de Ficte, tesorero y secretario del rey Carlos IX (fig. 3).

1576: el 8 de agosto es bautizado en Saint-Nicolas, el tercer hijo llamado Henry, en honor al antiguo protector Enrique II o tal vez a su hijo Enrique III. En el acto don Pedro es llamado por primera vez gentilhombre. El padrino, monsieur de la Poterie, actúa en nombre del nuevo rey Enrique III, elevado al trono en 1574 (fig. 4).

${ }^{10}$ Hasta ahora, en ausencia de certificados de bautismo se estimaba para los hijos las siguientes fechas de nacimiento: Paule (1572), Catherine (cerca 1575), Madeleine (nacida en 1573 o 1577), Françoise (entre 1579 y 1581), Henry (1580), Pierre (cerca de 1580), 


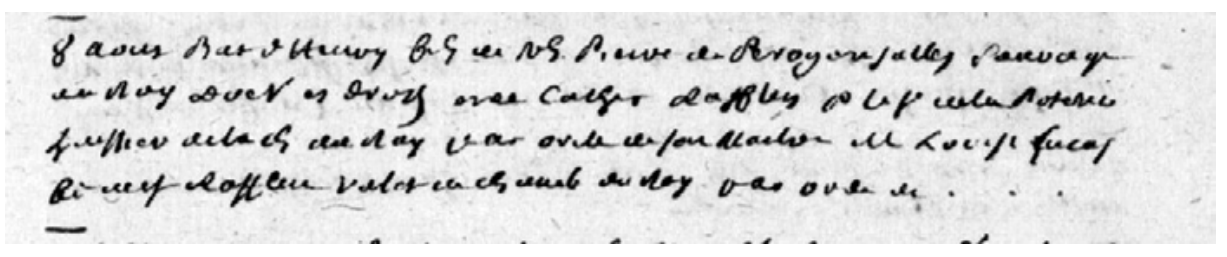

Fig. 4. «8 aoùt, Bat. (baptême) d'Henry, fils de N[oble] H[omme] Pierre de Perogonsalles, sauvage du Roy, docteur és droit, avec Catherine Rafflin. P[arrain] le sieur de la Poterie, huissier de la chambre du Roy, par ordre de son maitre; M. Louise Lucas, femme du sieur Rafflin, valet de chambre du Roy, par ordre (en blanc)».

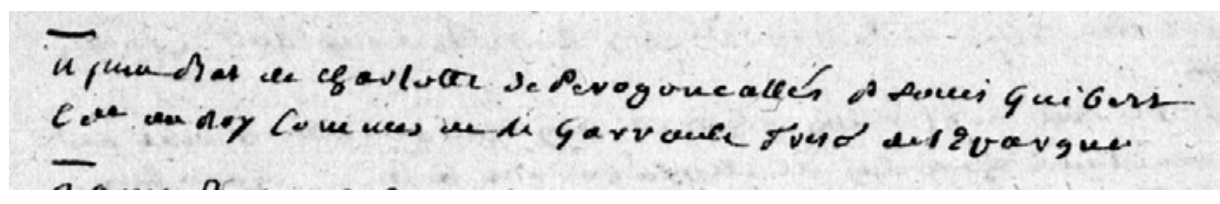

Fig. 5. «11 juin Bap (baptême) de Charlotte de Perogoncallés P[arrain] Louis Guibert conseiller du roy commis de M. Garrault trésorier de l'Epargne».

1578: el 11 de junio es bautizada su cuarta hija, Charlotte, y el padrino es uno de los consejeros del rey, Louis Guibert (fig. 5).

Estos datos ayudan a conocer la figura don Pedro como hombre y padre de familia. Sin embargo, es en su vida pública donde los nuevos documentos aportan las informaciones más relevantes, porque demuestran que no solamente no se escondió para ocultar la «vergüenza» de sus pelos y su «diversidad», sino que ocupó posiciones de gran relevancia en París:

1562: gracias a una pensión real de 70 liras anuales, estudia derecho en la universidad de Poitiers, donde se forma en derecho. La universidad de Poitiers era la segunda universidad de Francia después de la de París.

1571: es marguiller de la iglesia de Saint-Nicolas du Chardonnet, o sea miembro del consejo de laicos encargados de administrar las propiedades de la parroquia.

1571: en un acto es indicado como lecteur ordinaire du roi, el joven rey Carlos IX, quien entonces tenía veinte años. El lecteur era el cortesano encargado de leerle al rey los libros útiles para su diversión e instrucción y que, por esta razón, gozaba del privilegio de su constante frecuentación.

1582: Don Pedro es doctor en Derecho Canónico y profesor de esta disciplina en una universidad de París, tal vez la faculté de décret de la Sorbona ${ }^{11}$.

${ }^{11}$ Comunicación personal de Isabelle Bouillot que esta por publicar una biografía de don Pedro y de su familia. 


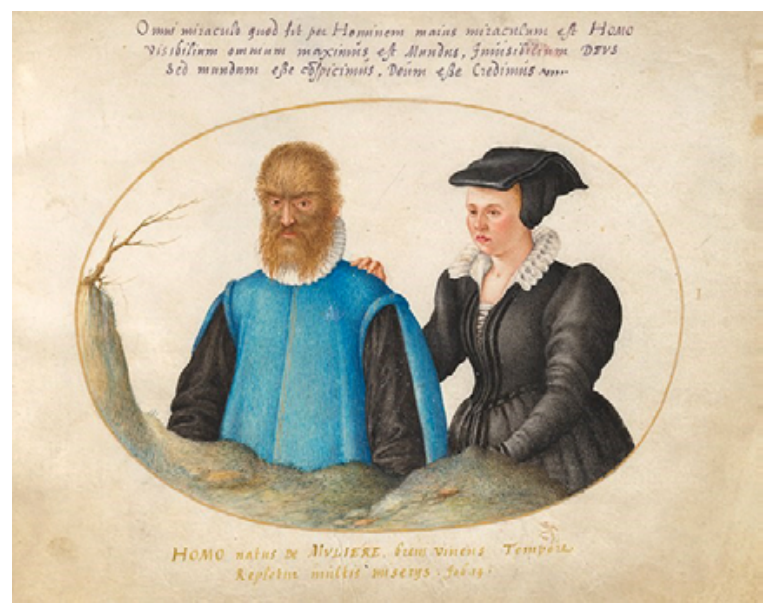

Fig. 6: Don Pedro y Caterina: Joris Hoefnagel-Washington, National Gallery of Art.

Como demuestran estos documentos, el joven Pedro que en 1560 era «sommelier», un simple sirviente en la mesa del soberano, en pocos ańos se había transformado en un distinguido gentilhombre, un doctor en derecho encargado de administrar los bienes de una importante parroquia de París, que tenía acceso privilegiado a la presencia del rey en calidad de su lecteur y que había llegado a la posición de profesor de Derecho Canónico en la más prestigiosa Universidad de Francia, la Sorbona.

\section{EL VIAJE A ITALIA (1585-1618)}

Repentinamente, y sin una aparente razón, a comienzos de los años ochenta del siglo XVI, la relación privilegiada de don Pedro con la corte francesa parece interrumpirse y todo cambia en su vida y en la de su familia, como está atestiguado por tres acontecimientos:

1582: con la esposa y dos hijos deja París y viaja a Múnich en Bavaria ${ }^{12}$, tal vez en la búsqueda de un nuevo protector. En esta oportunidad, el ilustrador Joris Hoefnagel, por encargo del duque de Bavaria, Guillermo V, realiza dos pequeños retratos de la familia Gonzales que introduce en una obra dedicada al reino animal y vegetal. En una de ellas aparece Don Pedro en compañía de su esposa Catherine (fig. 6).

12 Comparavit Monachii boiorum a[nn]o 1582. En Joris Hoefnagel: Animalia Rationalia et Insecta (Ignis), Plate I, Washington, National Gallery of Art. Algunos biógrafos dudan que este viaje haya tenido realmente lugar. 
1484: Don Pedro y Catherine se encuentran nuevamente en París, venden la casa de Saint-Victor y se mudan a Féricy-en Brie, lejos de la corte ${ }^{13}$.

1485: Catherine, con los hijos Henry y Charlotte, viaja a Basilea, Suiza, donde encuentran al doctor Felix Platter. Afortunadamente Platter nos ha dejado un relato de este viaje: «La esposa (de don Pedro) y sus hijos, un varón de nueve años (Henry nacido en 1476) y una muchacha de siete (Charlotte nacida en 1478) fueron enviados al duque de Parma en Flandes con destino Italia» ${ }^{14}$.

Por cierto, no sabemos por qué don Pedro no habría viajado con su esposa a Basilea y tampoco sabemos por qué motivo los Gonzales fueron enviados a Alessandro Farnese, duque de Parma y Gobernador de Flandes por cuenta del rey de España Felipe II. Lo cierto es que, a pesar de sus éxitos, evidentemente no era un hombre totalmente libre y no podía reusarse a obedecer las órdenes de sus protectores, aunque esto quisiese decir abandonar su vida y su segunda patria, Francia.

Después de la aparición en Basilea con rumbo a Italia, no tenemos mayores noticias de los Gonzales ${ }^{15}$ por ańos. Solo en mayo de 1591 unos documentos registran la presencia de don Pedro con su esposa y una hija en Parma, en la corte del duque Alessandro Farnese. Desde ese momento, y hasta la muerte en 1618 a los ochenta años, su nuevo país de residencia sería Italia. Una exhaustiva biografía del período italiano de Don Pedro y de sus hijos se encuentra en «Il selvaggio gentiluomo» de Roberto Zapperi:

1592: el 13 de agosto de 1592 en Parma, Catherine da a la luz un hijo peludo, Orazio, que tuvo como padrino de bautismo a Ranuccio Farnese, hijo de Alessandro y futuro duque de Parma.

${ }^{13}$ Después de esta fecha no se han encontrado otros documentos que certifiquen su presencia en Francia.

14 «Ex horum numero (de los hirsutos) Lutetiae erat vir quidam, ob raram pilositatem totius corporis, Regi Henrico II percharus, $\&$ in illius aula versatus, prolixis admodum pilis totum corpus, faciemque omnino, si exiguam regionem sub oculis excipias, obsitam habens, supercilijs \& crinibus in fronte adeo longis, ut eos sursum, ne visu impedirent, premere cogetur. Hic uxore ducta glabra $\&$ alijis mulierubus simili, liberos cum ea procreavit, hirsutos quoque, qui Duci Parmensi in Flandriam missi fuerunt, quos in Italiam una cum matre, masculum $9 \&$ foeminam 7 annorum transportandos hic Basilee vidi, Anno 1583 \& depingendos curavi. Erant facie hirsuta, magis masculus, minus paulo puella, cuius tota regio secundum spinae dorsi longitudinem, prolixis admodum pilis erat hispida». Felix Platter: «Observationum Felicis Plateri libri tres», Basila (1653) pp. 572-573. Felix Platter indica como fecha del viaje el 1583. Sin embargo, esta fecha no es aceptable porque no corresponde a las edades de Henry y Charlotte. Ha sido notado que también en otras oportunidades Platter se equivoca en indicar las fechas de los acontecimientos narrados por él.

15 Tal vez fueron a vivir por un tiempo en Alemania o en Austria, donde su pilosidad había despertado gran curiosidad en los años anteriores. Entre las familias nobles que se habían interesado a los Gonzales hay Guilllermo V duque de Bavaria, Carlo de Asburgo y, sobre todo, el archiduque Ferdinando. 
1592: Don Pedro que, en sus palabras, «no quería comer pan de gorra $»^{16}$, es nombrado administrador de una granja de los Farnese en Collecchio, a unos pocos kilómetros de Parma.

1595: el 27 de abril nace su último hijo, Ercole, también peludo.

1608: abandona Parma y se muda a Capodimonte (Nápoles) con toda su familia, a la casa de su hijo Enrico, protegido del Cardenal Odoardo Farnese.

Cerca de 1618, don Pedro el Selvaggio, como fue llamado durante su permanencia en Italia, muere a la edad de ochenta años al lado de Caterina, la compañera de toda una vida, quien fallecería en 1623.

\section{CONCLUSIONES}

Como consecuencia de su rara enfermedad don Pedro Gonzales tuvo una vida de enfrentamientos con los juicios y prejuicios de sus contemporáneos. Hubo quien vio en él al gentilhombre, al cortesano, al hombre culto integrado socialmente, y quien lo consideró solo un salvaje, un hombre-lobo.

Esta disparidad de opiniones se corresponde con la percepción que se tenía en el siglo xvi de la deformidad, que en muchos casos era todavía sinónimo de monstruosidad, de alteración del orden general establecido por la naturaleza.

La historia de don Pedro Gonzales, por lo tanto, va más allá de su experiencia personal, porque representa uno de los primeros intentos de las sociedades europeas de superar las convenciones socialmente aceptadas sobre lo que debía considerarse «normal» $»^{17}$.

Con sus éxitos sociales y académicos, don Pedro, sin quererlo, cuestiona el significado negativo que se atribuía a la deformidad e introduce a las sociedades europeas en la modernidad. Con él la "diversidad" deja de ser solo un motivo de exclusión social y empieza a ser considerada como una de las muchas posibilidades que existen hoy para definir «un ser humano».

Pero ¿qué pensaba don Pedro de sí mismo y de su extraña experiencia? Por suerte nos han llegado unas pocas líneas escritas por él en 1582 en que el sauvage $d u$ roi sintetiza la historia de su vida ${ }^{18}$ :

16 Zapperi, R. op. cit., p. 78.

17 Pentassuglia, P. op. cit.

18 «Petrus Gonsalus alumnus regis Gallorum, ex insulis Canariae ortus. / Me Teneriffa tulit, villos sed corpore mirum naturae. Gallia, mater / altera, me puerum nutrivit adusque virilem / aetatem: docuitque feros deponere mores / ingenuasque artes, linguamque sonare Latinam. / Contigit et forma praestanti munere divum / coniunx et thalami charissima pignora nostri. / Cernere naturae licet hinc tibi munera: nati / quod referunt alii matrem formaque colore, / ast alii patrem vestiti crine sequuntur» "Comparavit Monachii boiorum a[nn]o 1582». Marisa Anne Bass: Insect Artifice: Nature and Art in the Dutch Revolt, Princeton University Press (2019), p. 270, nota 60. Esta autobiografía se ha encontrado escrita en el verso de la miniatura dibujada por Hoefnagel por el duque de Bavaria Guilllermo V (fig. 6). 


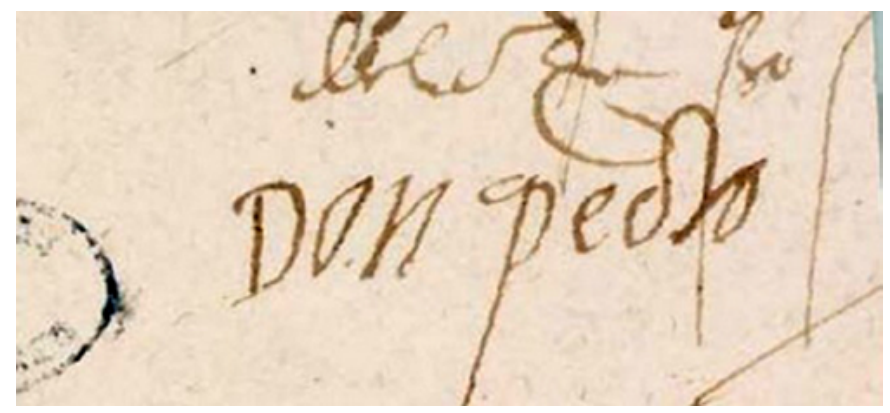

Fig. 7: firma de Don Pedro Gonzales.

Pedro Gonzales, criado por el rey de Francia, nacido en las Islas Canarias.

Tenerife me dio a luz y la obra admirable de la naturaleza me cubrió de pelos todo el cuerpo. Francia, mi segunda madre, me crío desde niño hasta que fui mayor y me enseñó a dejar mis costumbres salvajes, a aprender las artes liberales y a hablar en latín.

Por gracia divina me tocó en suerte una esposa de singular beldad y unos hijos, frutos preciosos de nuestra unión nupcial; puedes ver la generosidad de la naturaleza en el hecho que algunos hijos recuerdan la madre por la belleza y el encarnado otros hijos, en cambio, cubiertos de pelos, se asemejan al padre.

Como puede verse en esta autobiografía, don Pedro Gonzales agradeció a su segunda patria, Francia, por todo lo que de ella había recibido. Sin embargo, no se olvidó nunca de las Islas Canarias, sus raíces. No solamente siempre quiso que se le llamara «Pedro» en lugar de «Pierre», su nombre francés, sino que, con el nombre de «Don Pedro", firmó el único documento autógrafo que nos ha llegado" ${ }^{19}$ (fig. 7).

ReCiBido: 22-3-2021; ACEPTADO: 15-4-2021

19 Bouillot, I. Don Pedro, le "poilu» de la Renaissance, Historia n. ${ }^{\circ} 818$ (2015) pp. 72-76. 


\section{ANEXO 1}

1548- 17 de mayo

Procuration de Barthélemy Vallère, huissier de salle du roi, et ayant la charge de la dépense et nourriture du «sauvage du roi», à Pierre Hotman l'aîné, marchand orfèvre, bourgeois de Paris, pour recevoir ce qui lui sera dû à cause de ladite dépense, à commencer le ler juin, en bailler quittance à André Blondet, trésorier de l'épargne.

(Notaire Jean Trouvé - ANFr MC/ET/XIX/175)

1451- 1 de junio

Procuration donnèe par Francois de la Vacherie ayant la charge et gouvernement du saulvaige du Roy, n[otre] s[ire] à Pierre le Moyen, pour recevoir de M. André Blondet, trésorier de l'Epargne du roi les deniers qui luy sont ordennez par led. Sgr pour les moys de juillet, aoust et septiembre prochains venans pour la nourriture et gouvernement et despence du saulvaige d'icellui Sgr, qui est à raison de 50 sols. Par chacun jour, et ce pour l'absence dud. Constiutuant, qui s'en va au roiaulme d'Angleterre par le commandement du Roy et pour ses affaires:

(Maurice Roy: Artistes et monuments de la renaissance en France (1929) p. 352)

\section{2}

Je lis sur ceux de 1562, parmi les pensions régulières: «à Dom Pedro, sauvaige du Roy, escollier estudiant a l'Université de Poictiers, la somme de 77 livres pour la nourriture, despenses et entretenement de Luy, ses gens et chevaux»

(Arthur Heulhard «Villegagnon roi d'Amérique, un homme de mer au XVIe siècle 1510/1572», Ed. E. Leroux 1897, chapitre VV pp, 245-246)

1570- 2 de mayo

Bail pour 4 ans par Pierre de Gonzalès, dit Dom Pedro , sauvage du roi, rue Saint-Victor, d'une chambre dans une maison, rue Saint-Honoré, au prix de 66 sols t., jusqu'au jour de la Saint-Jean-Baptiste prochain, et à partir de la somme de 20 l.t. par an.

(Notaire Thomas Pèrier - ANFr MC/ET/XI/44)

1570- 9 de septiembre

Pierre de Perogonsalles dit "Dom Pedro", sauvaige et pensionnaire du Roy, demeurant à Paris et Catherine Rophelin, fille d'Anselme, marchand drapier, bourgeois de Paris, et de Caterine Pecan, épousa Pierre de Pero Gonzales, dit Dom Pedro, sauvage et pensionnaire du Roi, demourant a Paris.

Contrat de mariage: le pére donnait en dot la moitié d'une maison, sise rue Saint-Victor, entre la fleur de lys et le chef sainct Denys, a l'opposite du college Cardinal. Pierre de Perogonsalles fait donation à sa future épouse, dans le cas où elle lui survivrait sans enfants nés de leur futur mariage, de tous ses biens meubles et immeubles à l'exception de moitié d'une maison à Paris, rue Saint-Victor, dont il donne la jouissance viagère à Anselme Raphalin, marchand drapier, bourgeois de Paris, père de Cather ine Raphelin, et à sa femme.

Le future époux constituait, a titre de donaire, 1500 livres tournois

(ANFr - Châtelet de Paris. Y//104-Y//111. Insinuations Y 110 - fol. $465 \mathrm{~V}^{\circ}$

Ch. Binet: «La Correspondance historique et archéologique» vol. 2 Paris 1895, p. 144) 
«Ledict sieur Vacherie Gentil-homme avoit autrefois esté en ceste ville (Poitiers) compaignon d'estude du sauvaige du Roy»

(Liberge M. (doyen de la facultè de droit de Poitiers): Histoire et ample discours de ce qui c'est faict et passé au siège de Poictiers (de 1569), Lyon 1569 p. 52)

1570- 9 de julio

Contrato de matrimonio

"Catherine Raphelin ou Raffelin fille d Anselme marchand drapier bourgeois de Paris et de Catherine Pecan épousa Pierre de Pero Gonzalès dit Dom Pedro sauvage et pensionnaire du Roi demeurant à Paris. Le père donnait en dot la moitié d'une maison sise rue Saint Victor entre la fleur de lys et le chef sainct Denys à 1 en face du collège Cardinal et le futur il constituait à titre de douaire 1500 livres tournois»

(Notaires Martin Maheu et Antoine Desnos ANFr. Y110, fol. 265 v.)

(Ch. Babinet: La correspondance historique et archéologique (1895) vol. 2 pp. 143-144)

1570- 9 de septiembre

contrato de matrimonio

«Pierre de Perogonsalles dit "Dom Pedro, sauvaige et pensionnaire du Roy», demeurant à Paris et Catherine Rophelin: contrat de mariage par lequel Pierre de Perogonsalles fait donation à sa future épouse, dans le cas où elle lui survivrait sans enfants nés de leur futur mariage, de tous ses biens meubles et immeubles à l'exception de moitié d'une maison à Paris, rue Saint-Victor, dont il donne la jouissance viagère à Anselme Raphalin, marchand drapier, bourgeois de Paris, père de Catherine Raphelin, et à sa femme».

(ANFr - Châtelet de Paris. Insinuations Y//104-Y//111 - Notice n. 3173 fol. 465 Vº)

\section{1}

"Rue de Saint-Victor: Domp Petre, sauvaige du roi, taxé à quinze livres tournois dont recepte en a été faicte cy-devant. De laquelle a esté aucun chose reccu comme il appert par ledit roolle» ( COmpte du don de trois cens mil livres t., octroyé par la ville de Paris au feu roy Charles [IX]dernier décédé, en l'année MVc soixante-unze ; M. Francois de Vigny, le jeune, commis»)

1571- 30 de abril

Promesse de surseoir à une obligation par Pierre de Gonzalès, sauvage du roi, dit dom Pedro.

(Notaire Francois Raffin - ANFr MC/ET/XLIX/145)

1571- 25 de junio

Quittance de dom Pedro Gonzalès, lecteur ordinaire du roi, avec les autres marguilliers de l'église de Saint-Nicolas-du-Chardonnet, de la somme de 300 l.t. pour le rachat des 25 l.t. de rente.

(Notaire Catherine Fardeau - ANFr MC/ET/XXXIII/56)

1571- 2 de octubre

«2 oct[obre] Bat (baptême) de francoise Perogonsalés P[arrain] N[oble] h[omme] Alain Veau cons[eille]r rece[veur] des fin[snc]es à Paris»

(Acte paroissiaux de l'église Saint-Nicolas du Chardonnet-Cabinet de titres vol. 764 p. 177) Trascripción de Marie-Francoise Limon-Bonnet. 
1572- 20 de septiembre

«Insaisinetur nobilis vir Domnus Petrus Sauvaige (ces deux mots rayés) Pedro Silvester Regis, de domo predicta sibi vendita per dictum magistrum Petrum Le Brun, sita in vico Sancti Victoris, censiva Capituli, mediante summa XXV l. t. annui redditus emphiteotici sub conditionibus et clausulis appositis in litteram coram notariis Bardin et de la Fons, die XIII Martii anno 1568 passatis. Et solvat pro juribus vendarum XII den. Par : pro sorte principali III l. t. in toto XVIII l. t. XVI de gratia capituli (Arch. nat. LL158 f. 360)

(P. Le Vayer La Correspondance historique et archéologique, Volume 2, 1895 p. 112)

1572- 18 de julio

acte de vente de Gonzalès Pierre, docteur d'université , et Raffelin Catherine et Raffelin, Catherine

(Notaire Thomas Pèrier - ANFr MC/ET/XI/46)

1572- 16 de diciembre

Quittance de Pierre Gonzalès, noble homme, sauvage du roi, l'un des marguilliers de l'église Saint-Nicolas du Chardonnet.

(Notaire Thomas Pèrier - ANFr MC/ET/XI/46)

1572- 19 de diciembre

«19 xbe (décembre) Bat (baptême) de Perre Goncales P[arrain] Me Pierre de Ficte Cons[eille] r treso[rier] de l'Epargne»

(Acte paroissiaux de l'église Saint-Nicolas du Chardonnet - Cabinet de titres vol. 764 p. 177)

Trascripción de Marie-Francoise Limon-Bonnet

1573- 12 de noviembre

Titre nouvel par Pedro de Gonzalès, Sauvage du roi, docteur ès droits, rue Saint-Victor.

(Notaire Thomas Pèrier - ANFr MC/ET/XI/47)

\section{5}

titre nouvel Saint-Victor (rue) Gonzalès, Pierre, docteur d'université

(Notaire Thomas Pèrier - ANFr MC/ET/XI/48)

1576- 8 de agosto

«8 aoùt, Bat. (baptême) d'Henry, fils de N[oble] H[omme] Pierre de Perogonsalles, sauvage du Roy, docteur és droit, avec Catherine Rafflin. P. le sieur de la Poterie, huissier de la chambre du Roy, par ordre de son maitre; M. Louise Lucas, femme du sieur Rafflin, valet de chambre du Roy, par ordre (en blanc)"

(Acte paroissiaux de l'église Saint-Nicolas du Chardonnet (à laquelle appartenait rue de SaintVictor) - Cabinet de titres vol. 764 p. 179) Trascripción de Marie-Francoise Limon-Bonnet

1577- 11 de enero

Déclaration au sujet de la constitution de 25 l.t. de rente annuelle, par Pierre de Gonzalès, sauvage du roi, et Catherine Raffelin, sa femme.

Notaire Thomas Pèrier - ANFr MC/ET/XI/50 
1577- 11 de enero

acte de vente constitution de rente rue Saint-Victor pour Pierre Gonzalès et Catherine Raffelin,

(Notaire Thomas Pèrier - ANFr MC/ET/XI/50)

1578- 11 de junio

«11 juin Bap (baptême) de Charlotte de Perogoncallés P[arrain] Louis Guibert conseiller du roy commis de M. Garrault trésorier de l'Epargne»

(Acte paroissiaux de l'église Saint-Nicolas du Chardonnet-Cabinet de titres vol. 764 p. 180)

Trascripción de Marie-Francoise Limon-Bonnet

\section{2}

Don Pedro es doctor de Derecho Canónico y profesor de esta materia en una universidad de París

(Comunicación personal de Isabelle Bouillot)

1584- 20 de marzo

Bail à loyer par Pierre de Gonzalès, dit dom Pedro, sauvage du roi, pensionnaire du roi à Paris. (Notaire Thomas Pèrier - ANFr MC/ET/XI/58)

1584- 12 de abril

Pierre de Gonsales dit Sauvage et pensionné du roi à Féricy-en Brie. Vente et transport. Quittance.

(Notaire Thomas Pèrier - ANFr MC/ET/XI/58)

1584- 14 de abril

acte de vente cession en Portugal pour Pierre Gonzalès

(Notaire Thomas Pèrier - ANFr MC/ET/XI/58)

1584- 30 de mayo

«Insaisinetur Gabriel Le Maistre et Simone Legras, ejus uxor, de certa curia et pertinentibus consistentibus in duobus corporibus domorum, per ballium amphiteotici tradita Petro Gonzallez, dicto Pedro Sylvestri, acquisita mediante M III XXXIII 1/3 cum XXV 1.t. anconclusionem diei nui redditus debiti Collegium Bonorum Infantium, prout constitit litteris signatis Fardeau et Perier, de data diei XII aprilis 1584, et solvat pro qualibet libra XII den p. juxta conclusionem diei XIII aprilis (Ibid LL164 f. 282)»

(P. Le Vayer La Correspondance historique et archéologique, Volume 2, 1895 pp. 111-112)

1584- junio

Bail à loyer par Pierre de Gonzalès, dit dom Pedro, sauvage du roi, pensionnaire du roi avec son beau-frère et sa belle-soeur, de une maison à deux corps, cour et jardin au faubourg Saint-Marcel.

1584- 20 de junio

Vente d'une maison en rue Saint-Victor par Pierre de Gonzalès dit dom Pedro, sauvage et pensionnaire du roi qu'il avait fait construire. Contrat signé Don Pedro.

(Notaire Thomas Pèrier - ANFr MC/ET/XI/58) 
1584- 28 de junio

Pierre de Gonzalles, sauvaiges pensionné du Roy, et Catherine Rafflin sa femme, demeurant en Féricy-en Brie.

(Notaire Cathereau - Cabinet de Titre)

(Hercé, L'Intermédiaire des chercheurs et curieux (1888) p. 613)

1584- 13 de septiembre

Daulphin Charpentier, prêtre, vicaire de l'église Saint-Gervais, confesse avoir reçu de Pierre Gonzalès dit dom Pedro, sauvage du roi, pensionnaire du roi, la somme de 145 écus d'or sol, pour le rachat de 8-1/3 écus de rente annuelle.

(Notaire Thomas Pèrier - ANFr MC/ET/XI/59) 


\section{ANEXO 2}

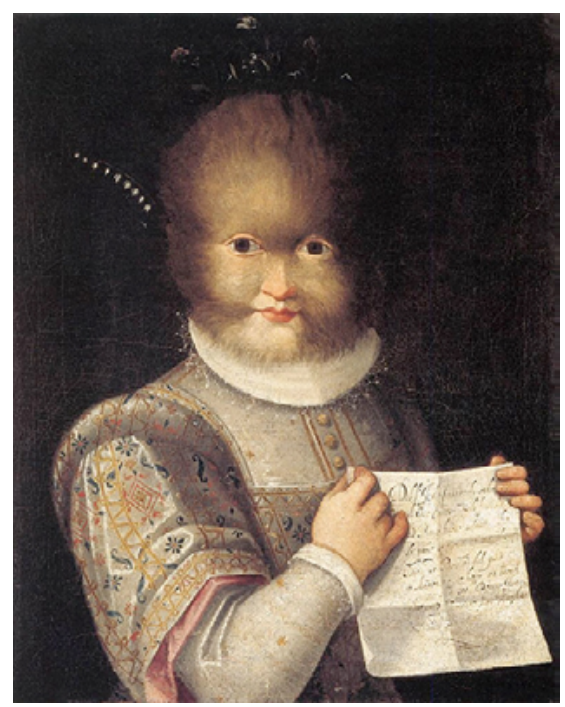

Retrato de Antonietta Gonsalvus (Tognina) por Lavinia Fontana (Château de Blois).

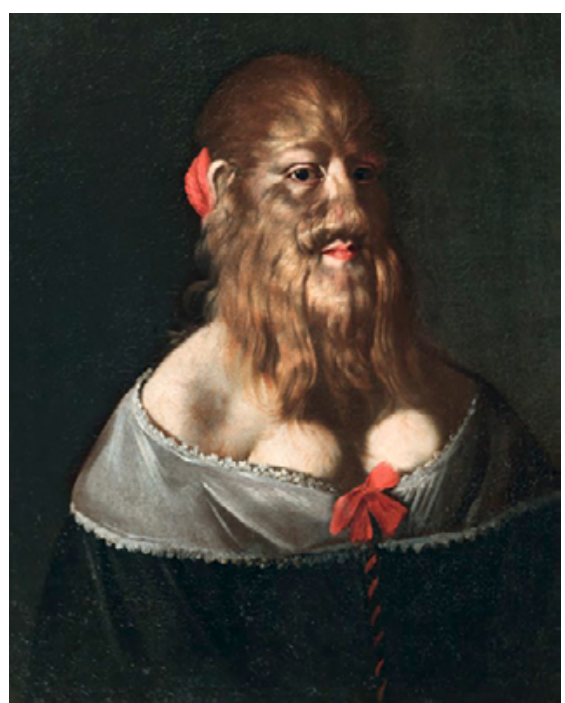

Retrato de escuela napolitana (1610-1630) de una de las hijas de don Pedro Gonzales, tal vez Tognina. (colección privada). 
